\title{
Stabilization and extinction mechanisms of flames in cavity flameholder scramjets
}

\author{
Jiangheng L. Ruan, Guillaume Ribert and Pascale Domingo \\ CORIA - CNRS, Normandie Université, INSA de Rouen Normandie \\ 76000 Rouen, France
}

ARTICLE HISTORY

Compiled October 23, 2020

\begin{abstract}
Stabilizing a flame in scramjet engines is a current technological challenge. Indeed, the residence time in the combustion chamber is very short, thus limiting the mixing efficiency and consequently, the combustion. This problem is presently addressed by simulating the scramjet combustor of the U.S Air Force Research Laboratory in which ethylene is injected from the back of a slanted cavity to react with the supersonic airflow. Large-Eddy Simulations (LES) are performed for different cases showing a dependence of the combustion regimes encountered in the scramjet cavity with the fuel flow rate. For a medium-high flow rate, a mix of lean and rich premixed reaction zones is found along with non-premixed flames. At medium flow rate, rich premixed flames disappear but the flame remains stable in the cavity. The mechanism of flame stabilization is detailed and the LES tool has proven its efficiency in such challenging configuration. Finally, a mechanism of extinction is proposed when the fuel flow rate is further reduced.
\end{abstract}

\section{KEYWORDS}

Supersonic combustion; Large-eddy simulation; Cavity-based scramjet

\section{Introduction}

Scramjet engines are good candidates for hypersonic flight applications [1, 2]. However, at such high speeds, the residence time of air and fuel in the combustor becomes so short that the mixing and burning processes become ineffective if no recirculation zone is encountered. The problem of flame-holding, along with the understanding of the mechanisms of flame stabilization and flame extinction, are thus key elements in the development of a future reliable scramjet engine.

Among the different strategies to trap the fuel and air in recirculation zones [1, 2], the use of cavity has been viewed as a promising solution [3] since it reduces drag, lowers total pressure losses and minimizes aerodynamic heating [4]. Resolving problems related to cavity scramjets requires the treatment of various problems such as the effect of cavity geometry and fuel injection parameters which ultimately condition the impact of heat release on the structure and the dynamics of the shear layer above the cavity, the recirculation zones, the flame regimes or the conditions of flame blowout. The result is a complex flow-field that greatly complicates the identification of the flame-holding mechanisms and the prediction of flame stability. Nevertheless, among the various observations made on such device, it may be noted that inside 
the cavity, a primary vortex with a strong circulation is responsible for the mass exchange with the free-stream zone while a secondary vortex is supposed to act as a fuel pooling in a dead zone. In addition, the effect of heat release on the local flow field reduces the growth rate of the shear layer and increases the average residence time of the recirculating fluid relative to a non-reacting case. Finally, cavity blowout limits can be extended if the residence time increases, by changing the geometry of the cavity, increasing the stagnation temperature and pressure of the free-stream, or changing the location of the injector and its fuel rate.

It becomes then a formidable challenge to predict such behaviors and the use of numerical simulations appears as a necessary tool [5]. In particular, the use of Large-Eddy Simulation (LES) for supersonic combustion is now becoming relevant [6, 7, 8, 9, 10] even if for highspeed combustion, the choice of turbulent-combustion models can be challenging [11, 12] since the majority of models in literature have been developed for low-speed combustion. Besides, combustion can occur near or in the distributed-reaction-like regime [5], i.e. when high-speed reacting flows have also high levels of turbulent fluctuations, combustion regime that is difficult to model.

In this study the LES tool is used to reproduce the processes arising in the AFRL (Air Force Research Lab) combustor [13]. Experimental data are used to validate the numerical approach and support an analyze on the flame behaviors and regimes in the case of a stable and of a vanishing flame.

\section{Configuration and numerical set-up}

\subsection{Experimental cases}

The experimental configuration under study is a supersonic cavity flameholder [13]. It consists in a converging-diverging nozzle followed by an isolator that is $H=50.8 \mathrm{~mm}$ high and $L_{i s o}=177.8 \mathrm{~mm}$ long. The bottom wall of the test section has a divergence angle of $\alpha=2.5^{\circ}$, which begins at the end of the isolator. The cavity is located $76.2 \mathrm{~mm}$ downstream of the end of the isolator and has a depth of $D=16.5 \mathrm{~mm}$ with a floor length of $46.1 \mathrm{~mm}$ ( $L=66 \mathrm{~mm}$ length to the ramp midpoint). The width of the facility is constant with a value of

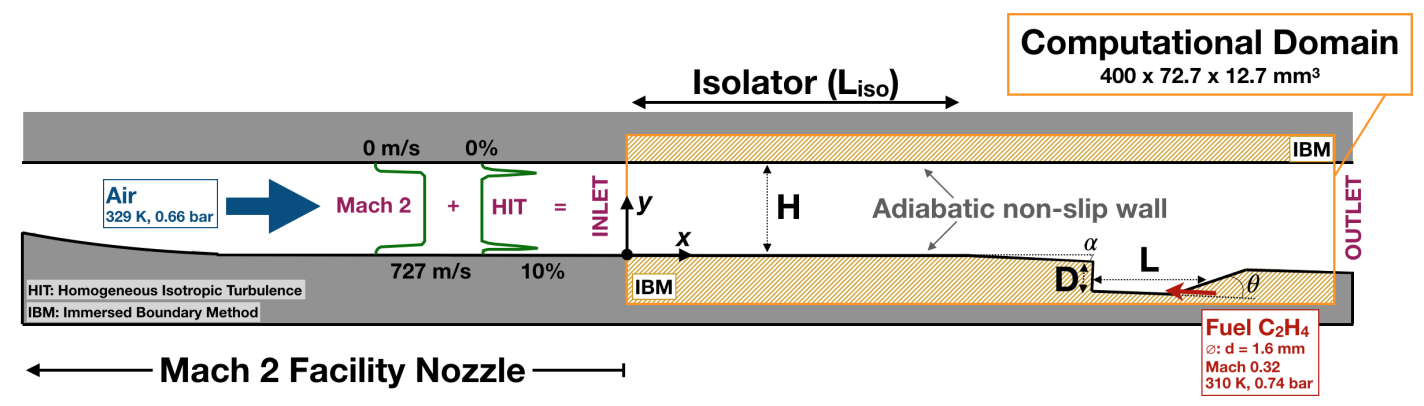

Figure 1.: Sketch of AFRL supersonic wind tunnel.

$152.4 \mathrm{~mm}$ (see Fig. 1 1 for details). An array of 11 fuel injection ports of diameter $d=1.6 \mathrm{~mm}$ are evenly distributed on the aft cavity wall, at $y=-19.57 \mathrm{~mm}$.

In the isolator, air is at Mach 2 and the total temperature and pressure are $589 \mathrm{~K}$ and $483 \mathrm{kPa}$, respectively. Ethylene $\left(\mathrm{C}_{2} \mathrm{H}_{4}\right)$ is injected at $310 \mathrm{~K}$ at the rear of the cavity which makes the auto-ignition impossible and necessitates the use of spark plugs to force ignition. Three fuel loadings [13] have been simulated (see Table 11). For the cases MHF (Medium-High Fuel) and MF (Medium Fuel), experimental data for velocity and mean fuel concentrations (only MF) are available inside the cavity [13, 14]. The flame is found stable and located inside the cavity 
contrary to the case LF (Lean Fuel) for which the flame extinguishes. Additional information

\begin{tabular}{cccc} 
Cases: & MHF & MF & LF \\
\hline SLPM & 99 & 56 & 36 \\
Flame & Stable & Stable & Extinction
\end{tabular}

Table 1.: Fuel loading in Standard Liters Per Minute (SLPM).

about the experimental configuration can be found in numerical studies [15, 16, 17].

\subsection{Numerical configuration}

The simulations have been performed with the CORIA in-house code, SiTCom-B [8, 18, 19 , [20]. The conservation equations of species mass fractions, with complex molecular transport properties, momentum and total sensible energy are solved in their fully compressible form over the structured mesh in a finite volume formulation resorting to a skew-symmetric-like scheme for the convective fluxes [21] and to a fourth-order centred scheme for the viscous and diffusive fluxes. An addition of second and fourth-order artificial dissipation terms [22, 23, 24] is used to overcome spurious oscillations [25]. Time is advanced with a fourth order RungeKutta scheme. Navier-Stokes characteristic boundary conditions are applied to describe the air and fuel inflows while the outflow uses a zero-gradient condition. An immersed boundary method (IBM) [26, 8] is used to model the solid surface of the wind tunel.

Unsolved subgrid-scale fluxes are modeled by the Germano closure [27]. Sub-grid Prandtl and Schmidt numbers are set to 0.9. Mixture diffusion coefficients for each species [28] are considered based on binary diffusion coefficients [29]. The upper and lower walls of the scramjet are modeled with an adiabatic non-slip condition and side sections use periodic conditions since only one injector is considered. A detailed chemistry containing $N_{s}=22$ species and 206 reactions [30] is used to describe the combustion of ethylene with air. Note that it was not possible [31] to obtain a stable flame using a kinetic scheme with fewer species such as the ten reactive species mechanism from Jachimowski [32]. A no-model assumption is used to compute the chemical source terms as in [8, 18]. It has been verified in [10, 12, 31] that these assumptions do not modify the results and scenarios described hereafter. The use of the quasi-laminar-chemistry approach has been found acceptable in [5] for small scramjet-like problems.

As in [15, 16, 17], the nozzle is not computed but the 3D simulations start at the beginning of the isolator. The computational domain is extended $81 \mathrm{~mm}$ after the cavity. At inlet boundary, the three components of velocity profile are given (in $\mathrm{m} / \mathrm{s}$ ) by $U=$ $1.22 U_{0}(y / H)^{1 / 7}(1-y / H)^{1 / 7}$ with $U_{0}$ set to $727 \mathrm{~m} / \mathrm{s}$ and corresponding to a Mach number of $2, V=W=0$. Turbulence in injected at the inlet to trigger the boundary layer development with an intensity of $10 \%$ of the local axial velocity and distributed evenly on the three components of the velocity.

The mesh resolution uses 45 millions of cells, which are constant in $x$-direction $(\Delta x=$ $200 \mu \mathrm{m})$ but are refined near the wall and the mixing layer in $y$-direction $(\Delta y \in[150-200] \mu \mathrm{m}$ and $\Delta z \in[160-300] \mu \mathrm{m})$. Near the injector the mesh refinement in $y$-and $z$-direction leads to 10 the number of cells to describe the diameter of the injector. A more refined mesh using 300 millions of cells has been used to validate the procedure and analysis [31]. A wall-law model based on zero-equation mixing length model and using the van Driest damping function [33] is applied at the upper and lower wall of the simulation domain since the dimensionless wall distance $y^{+} \approx 10$. In the cavity, $y^{+} \approx 1$. Statistics have been gathered over $6 \mathrm{~ms}$ while the empirically estimated cavity residence time [34] is $0.9 \mathrm{~ms}$. 


\subsection{Validation}

Figure 2 shows the average fields of $\mathrm{C}_{2} \mathrm{H}_{4}$ and $\mathrm{O}_{2}$ mass fractions, heat release rate (HRR) and temperature at the injector centerplane for a medium fuel (MF) and medium high fuel (MHF) loading. HRR fields indicates that a strong combustion occurs in the mixing layer developing

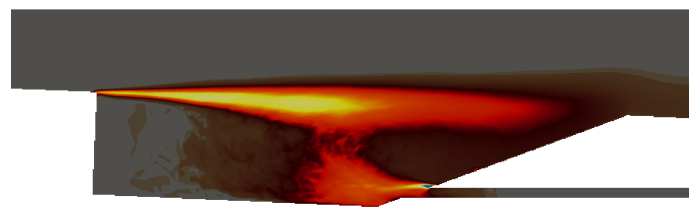

Heat Release Rate (GW/m3)

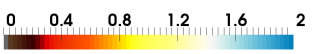

(a) Heat release rate (MF)

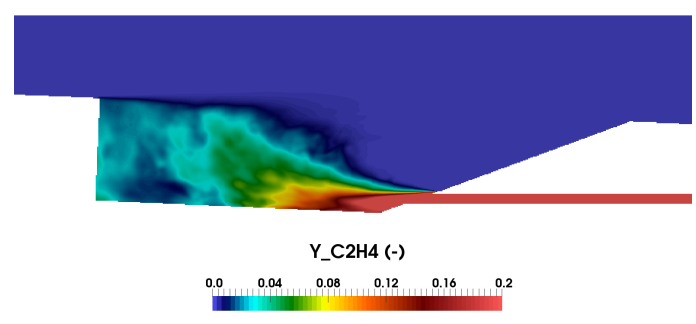

(c) $Y_{C_{2} H_{4}}(\mathrm{MF})$

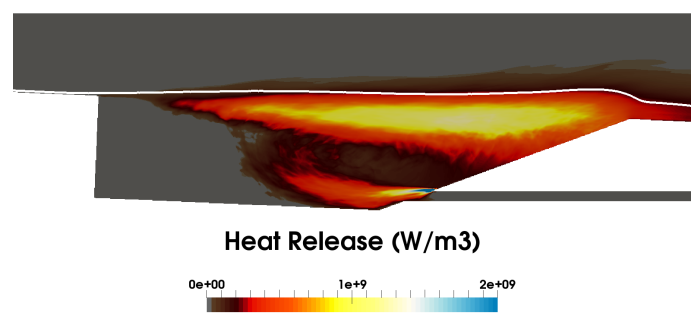

(e) Heat release rate (MHF)

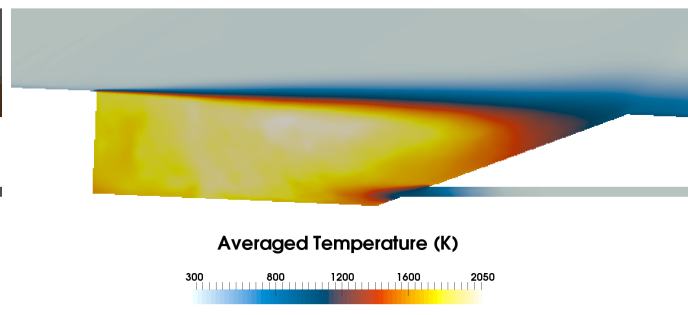

(b) Temperature (MF)

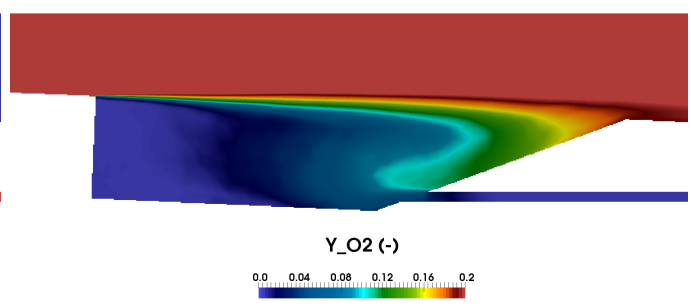

(d) $Y_{\mathrm{O}_{2}}(\mathrm{MF})$

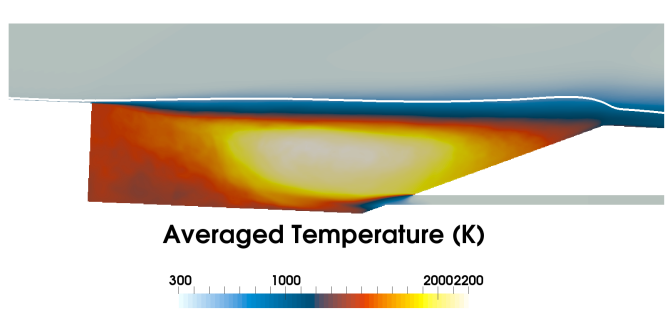

(f) Temperature (MHF)

Figure 2.: Average fields of $\mathrm{C}_{2} \mathrm{H}_{4}$ and $\mathrm{O}_{2}$ mass fractions, heat release rate and temperature at the injector centerplane for MF and MHF loading.

at the beginning of the cavity and at the exit of the injector for the case MF, but mainly above the ramp of the cavity and at the exit of the injector for the case MHF. Inside the cavity, an average gas temperature is found around $1800 \mathrm{~K}$ for both cases. A species stratification is observed. In particular, oxygen is absent at the front of the cavity, filled with ethylene and combustion products, but is present near the injector. Averaged streamlines colored by axial velocity are shown in Fig. 3. Both primary and secondary recirculation zones can be clearly identified. The fresh air feeds the cavity with the large recirculation zone, while the smallest one located upstream traps hot products mixed with unburnt ethylene. Comparisons between the numerical solutions and the experiment are displayed in Fig. 4 for the velocity profiles taken at $x=2,11,20,30,39,48,57,66 \mathrm{~mm}$ from the cavity front corner. The comparison of velocity profiles shows only a slight difference between the experiment and the numerical data for streamwise velocity. The discrepancies are located in first planes $(x=2$ and $11 \mathrm{~mm}$ ), in the mixing layer. The mixing layer in the experiment is thicker just behind the 


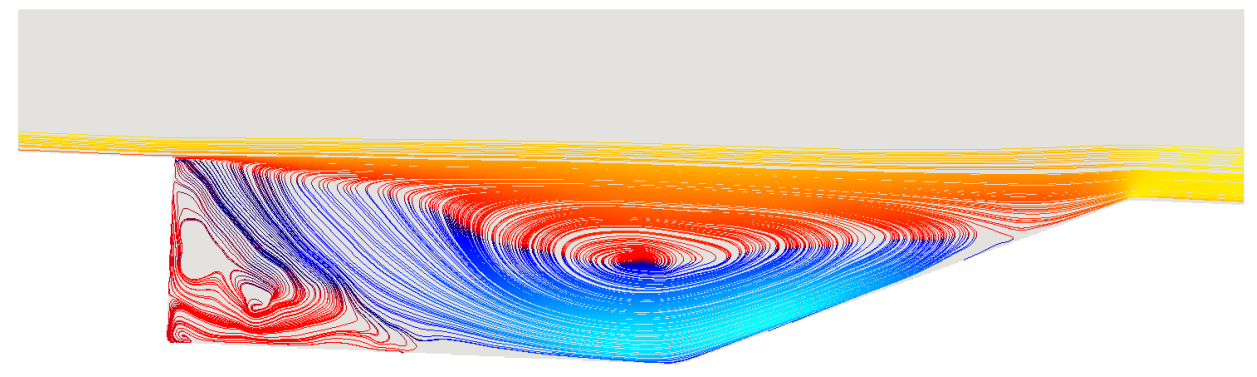

Averaged Velocity $X(\mathrm{~m} / \mathrm{s})$

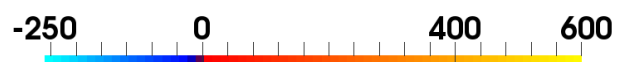

Figure 3.: Averaged streamlines, colored by averaged axial velocity (case MHF).

sudden expansion area, but the correct thickness is recovered further downstream. A better description of the mixing layer above the cavity would certainly have required a specific simulation of the nozzle [35, 36] out of scope of this study. The transverse velocity (Fig. 4) shows significant discrepancies between LES predictions and experimental measurements inside the cavity. One may remark that in the work from Hassan et al. [17], the transverse velocity has a similar behavior as in the present paper. The question is open on whether those discrepancies are related to modeling issues or to experimental uncertainties due to the difficulties encountered by PIV in a cavity. The equivalent fuel concentration $\left(\mathrm{X}_{C_{2} \mathrm{H}_{4}, e q}\right)$ in Fig. 5 is defined as $X_{C_{2} H_{4}, e q}=\sum_{i}^{N_{s}} K_{i} X_{i}$, with $X_{i}$ is the molar fraction of species $i$, which is defined as $\mathrm{C}_{n(i)} \mathrm{H}_{p(i)} \mathrm{O}_{q(i)}$ with $\mathrm{C}, \mathrm{H}$ and $\mathrm{O}$ the atoms of carbon, hydrogen and oxygen respectively, and where $K_{i}=p(i) / p\left(C_{2} H_{4}\right)$. Fairly good results are observed for the centerplane of the injector at $x=8.9 \mathrm{~mm}$ and $x=44.5 \mathrm{~mm}$. Overall, the trend is recovered showing a penetration of the fuel inside the cavity up to its front where the fuel is trapped. All these results are consistent with other literature articles for which RANS (Reynolds Average Navier-Stokes) [16] or different RANS-LES hybrid approaches [17] have been tested.

Further comparisons were made on the velocity and wall pressure profiles for the MHF case and a good agreement was found by comparison with the experimental data [10, 12].

\section{Flow analysis}

\subsection{Stabilisation mechanisms and combustion regimes}

The goal of the cavity is to increase the residence time and favor combustion, this effect being also present in the cavity employed in a subsonic combustor, as discussed in a recent review paper from Zhao et al. [37]. Note that it would be legitimate to wonder how the impact of the cavity is modified if the air inlet becomes subsonic (out of scope of the present study). The mechanism might not be that different that the one described below, except that the mixing layer would be thicker and the mixing would be more efficient.

In our study, a stable flame is found experimentally for both fuel loadings of 99 and 56 SLPM. This feature has been recovered by the present numerical simulations as shown in Fig. 2. For both cases, the whole cavity is at subsonic speed and two recirculation zones are created. For the case MHF (Fig. 6a), zone B features a large recirculation zone with high velocities (up to $350 \mathrm{~m} / \mathrm{s}$ ) and high temperature (up to $2500 \mathrm{~K}$ ) [31]. The temperature of the flow near the 


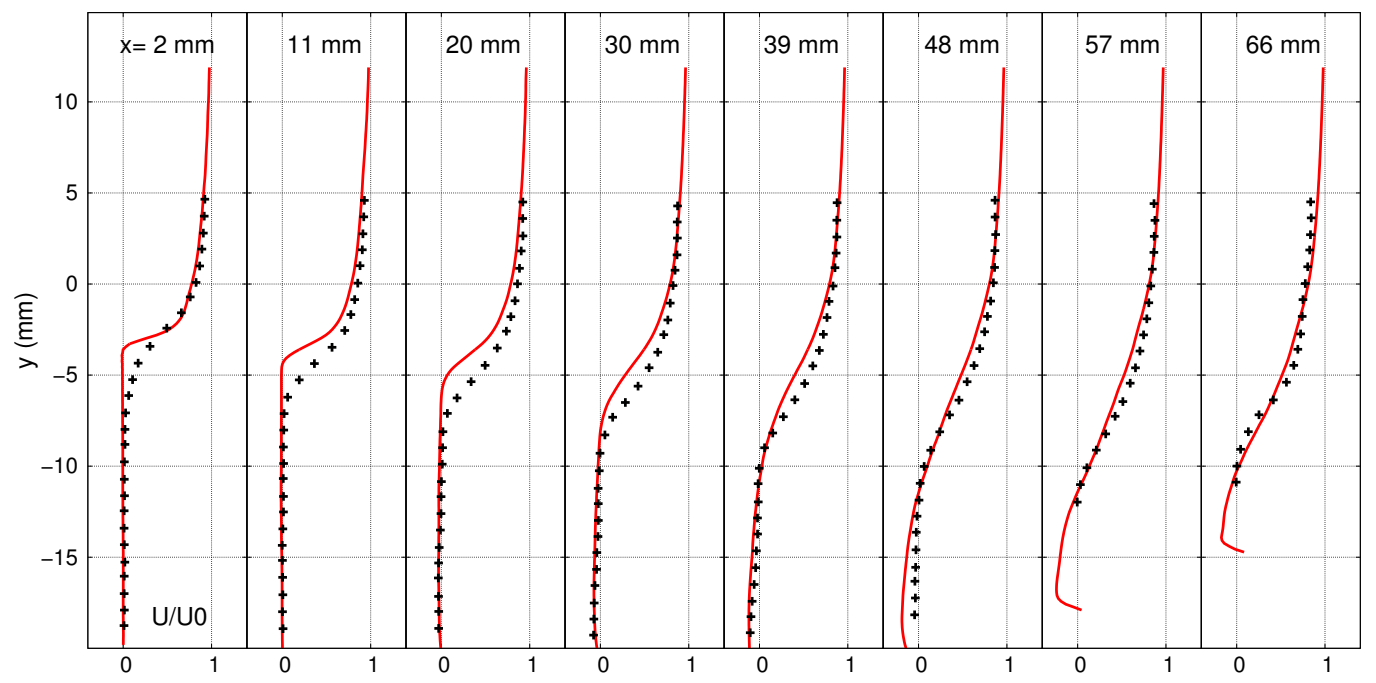

(a) Average streamwise velocity

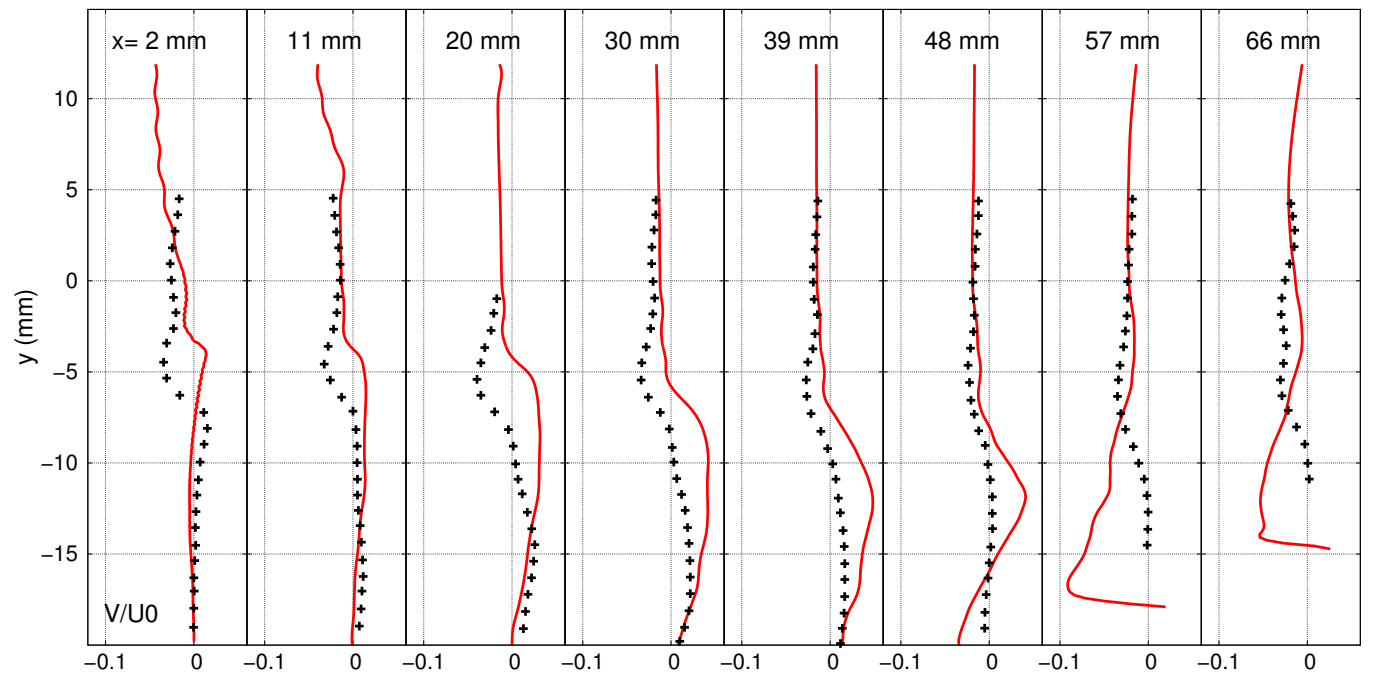

(b) Average transverse velocity

Figure 4.: Comparison between the numerical (lines) and the experimental (symbols [13, 14]) data inside the cavity. $(a, b)$ Centerplane between two injectors. Case MF.

cavity aft wall can reach $2000 \mathrm{~K}$ allowing the incoming airflow to be heated before encountering ethylene present in the cavity and thus support combustion. In zone A, i.e. at the front of the cavity, the flow velocity is one order of magnitude less than in zone B with a temperature below $1500 \mathrm{~K}$. The heat release rate snapshots (Fig. 6) show that the flame fronts are located at the exit of the injector, the interface between the two recirculation zones and the mixing layer between the cavity and the freestream. The isoline Mach $=1$ indicates that combustion occurs preferentially at subsonic speeds, i.e. inside cavity. The middle of the cavity and the interface between recirculation areas are driven by moderately strong positive and weak negative heat release rate respectively. Negative values of the heat release rate occur when the local mixture is in a state of over-equilibrium. As shown in Fig. 5 and 2(c), the fuel contained in the recirculation zone at the front of the cavity does not react because there is no more oxygen left. However at the top of zone A, hot ethylene (around $1500 \mathrm{~K}$ ) starts to react with the oxygen coming from the airflow in the mixing layer developing above zone $\mathrm{B}$. The strongest 

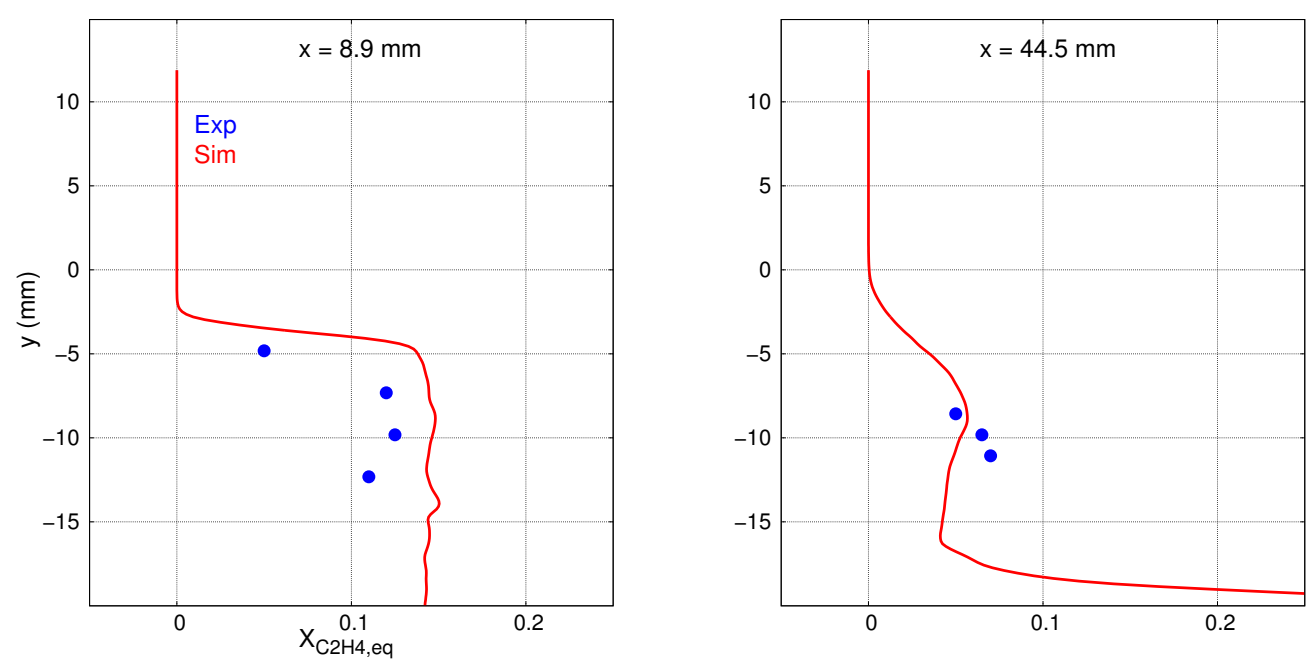

Figure 5.: Plots of $\mathrm{X}_{C_{2} H_{4}, e q}$ at two positions (case MF, injector centerplane). Bullets: experiment. Lines: LES.

combustion activity is in zone B, with the effect of heating the airflow coming from the back of the cavity. This hot air can then react with the fuel leaving the injector and the resulting combustion products will then feed the zones A and B with hot gases. This mechanism of recirculating hot and reactive gases allows to maintain a stable flame in the cavity.

For the case MF (Fig. 6b), the quantity of fuel going into zone A is smaller. The amount of fuel flowing from zone A to the mixing layer is also reduced compared to MHF. The fuel shortage in the mixing layer above the large recirculation area and near the cavity ramp is responsible of the low heat release rate present in this area. This results in high temperatures at the front of the cavity and the only "cold" area of the cavity is located at near the ramp as observed by Baurle [16] with RANS simulations.

To characterize the flame regimes, i.e. to distinguish premixed from non-premixed combustion, the flame index [38] is often used: F.I. $=\nabla Y_{F} \cdot \nabla Y_{O_{2}}$. A ponderation was further introduced by Lock et al. [39] to distinguish between lean and rich combustion:

$$
F . I .=\frac{1}{2} \frac{Z-Z_{s t}}{\left|Z-Z_{s t}\right|} \times\left(1+\frac{\nabla Y_{F} \cdot \nabla Y_{O_{2}}}{\left|\nabla Y_{F} \cdot \nabla Y_{O_{2}}\right|}\right) .
$$

In Eq. (11), $Z$ is the mixture fraction from the Bilger et al.'s expression [40] and $Z_{s t}$ the stoichiometric mixture fraction. $Y_{\mathrm{O}_{2}}$ is the oxygen mass fraction while $Y_{F}$ is defined presently as $Y_{F}=Y_{C_{2} H_{4}}+Y_{C_{2} H_{2}}+Y_{C H_{3}}+Y_{C_{H_{4}}}+Y_{C_{2} H_{6}}+Y_{C_{3} H_{6}}$. Eq. (1) is then used to identify the regimes of combustion: F.I. $=0$ for non-premixed flames, +1 for rich premixed flames and -1 for lean premixed flames. The flame index shown in figures 7 and 8 is only computed in zones where the heat release is greater than $1 \%$ of its maximum value. For the case MHF (Fig. 7), non-premixed flames are observed in the mixing layer above zone A, between zone $\mathrm{A}$ and $\mathrm{B}$ and near the middle of the ramp where an excess of carbon species react with an excess of hot air. Rich premixed flames are found in the mixing layer above zone B and in the region close to the middle of the cavity. Lean premixed flames are located near the cavity ramp. The regions with negative heat release rate (see Fig. 6) located at the interface between the recirculation zones or at the exit of the injector are only featuring rich premixed combustion regime. Neither lean premixed nor nonpremixed flames can be found with negative heat release rate. Furthermore, not all non-premixed regions are crossed by the stoichiometric line, the cavity is provided with rich non-premixed combustion near its center and lean non- 

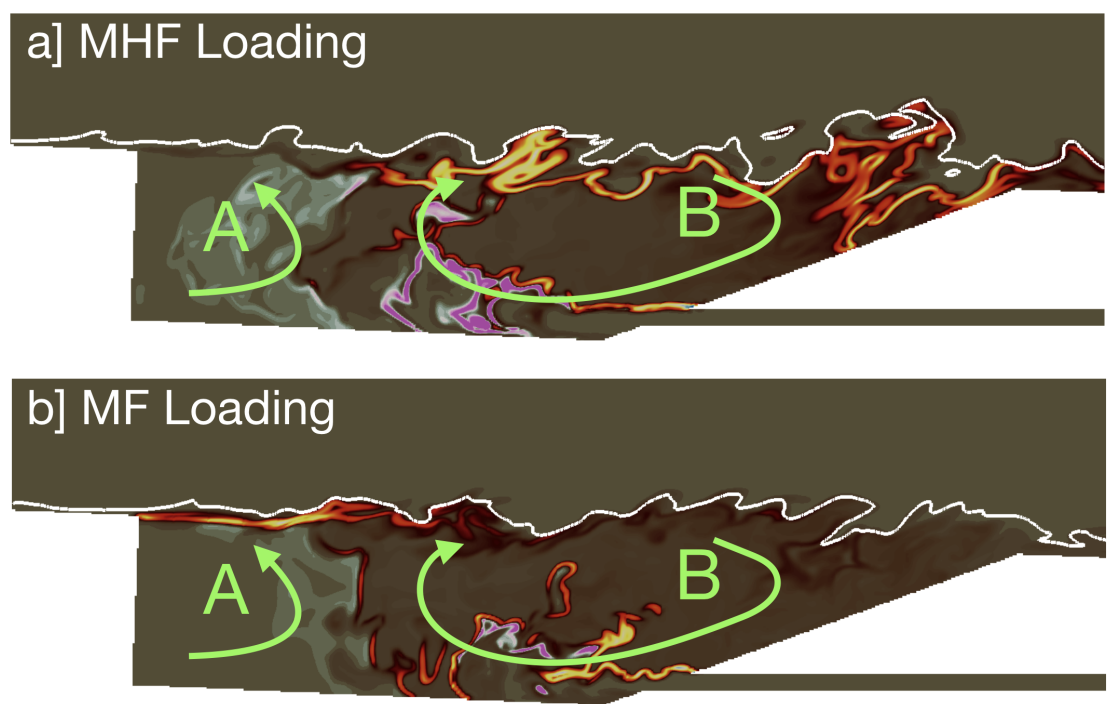

\section{Heat Release (W/m3)}

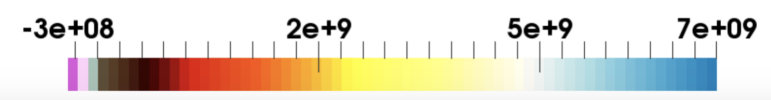

Figure 6.: Instantaneous fields of heat release rate taken at the injector centerplane. At the top, case MHF (a); at the bottom, case MF (b). White line: isoline Mach $=1$.

premixed combustion at its rear.

The flame index has also been computed and displayed in Fig. 8 for the medium fuel loading case (MF). The cavity is found to be mainly driven by non-premixed flames. All the regions with significant heat release rate (see Fig. 6) are featuring flame index values of 0, corresponding to non-premixed combustion. Rich premixed regime is only observed at the exit of the injector and barely at the mixing layer above the small recirculation area. The mixing layer above the large recirculation area and the cavity ramp are still controlled by premixed combustion, but only lean mixtures can be found in these zones. A lower injection velocity creates less turbulence in the cavity which makes the mixing more difficult.

Fig. 9 shows the conditional mean of heat release rate by flame index $<\dot{\omega}_{E} \mid$ F.I. $>$ adimensionalized by the overall mean heat release $\left\langle\dot{\omega}_{E}\right\rangle$ (blue patterns) and the contribution of each flame regime as characterised by the flame index to the heat release, $C_{F I}$ (red histogram) defined as follow:

$$
R_{F I}=\frac{<\dot{\omega}_{E} \mid F . I .>}{<\dot{\omega}_{E}>} \quad \text { and } \quad C_{F I}=R_{F I} \frac{N_{F I}}{N_{T}}
$$

with $N_{T}=\sum_{F I} N_{F I} . N_{F I}$ is the number of points in the interval of considered flame index and $N_{T}$ the total number of points. The conditional mean heat release rate is similar for each flame index value: from $R_{F I}=0.8$ in the nonpremixed flames to $R_{F I}=1.1$ in the premixed flames regardless of the mixture. None of the regimes is only marked by either strong or weak combustion. The values taken by $C_{F I}$ indicate that, for the case MHF, most of the contributions to the overall heat release rate come from premixed flames, over $75 \%$, where the lean premixed regime contribution is approximately $60 \%$. For the case MF, the premixed regime corresponds to $40 \%$ almost entirely dominated by the lean side.

The decrease in fuel loading has then a significant impact on the combustion regime which in turns impacts the choice of a combustion modeling if either a tabulated approach of the 


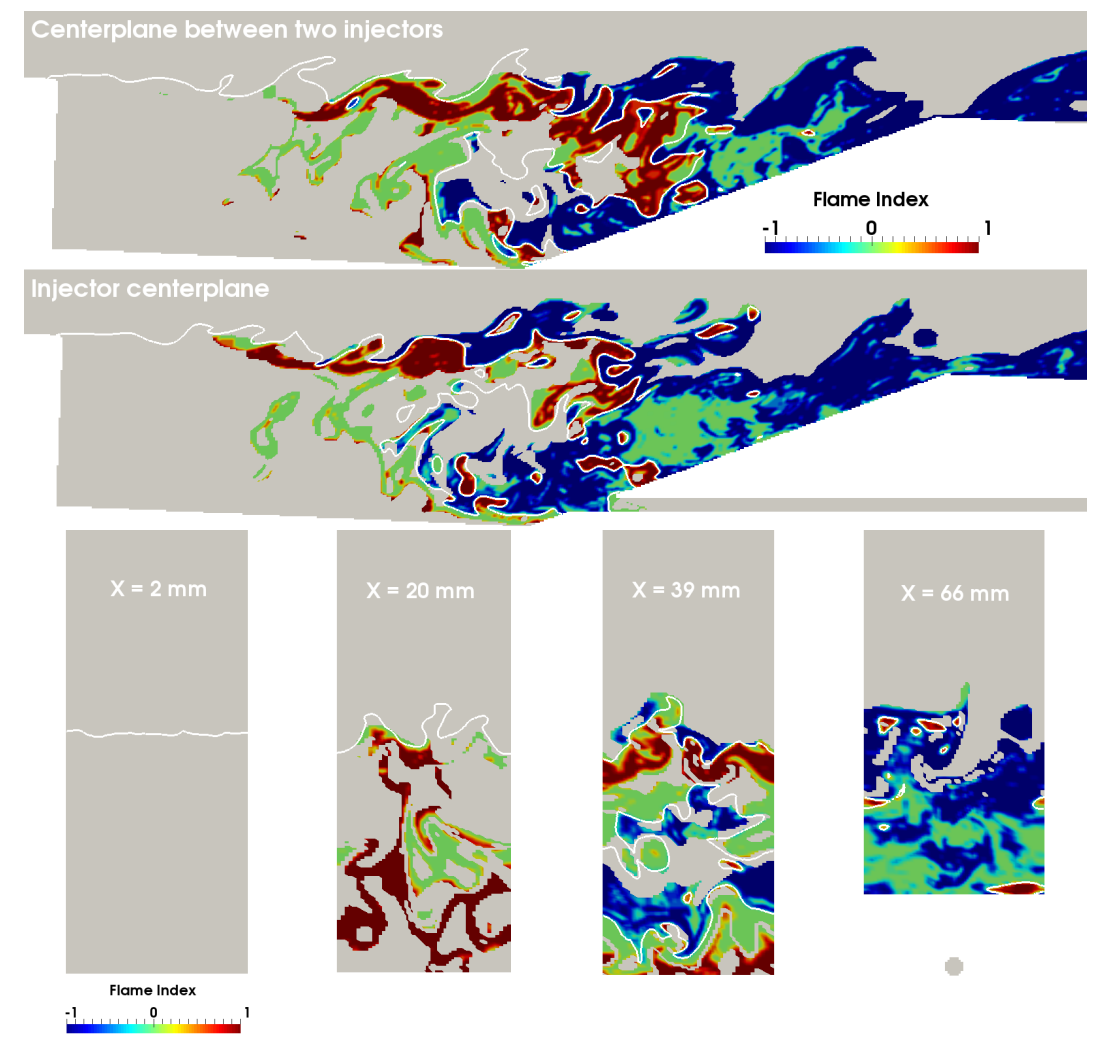

Figure 7.: Instantaneous flame index in the centerplane between two injectors, in the injector centerplane and in four planes with constant $x$ in the cavity. -1 : lean premixed, 0 : nonpremixed, 1: rich premixed. The isoline $Z=Z_{s t}$ is displayed with a white line. Case MHF. 

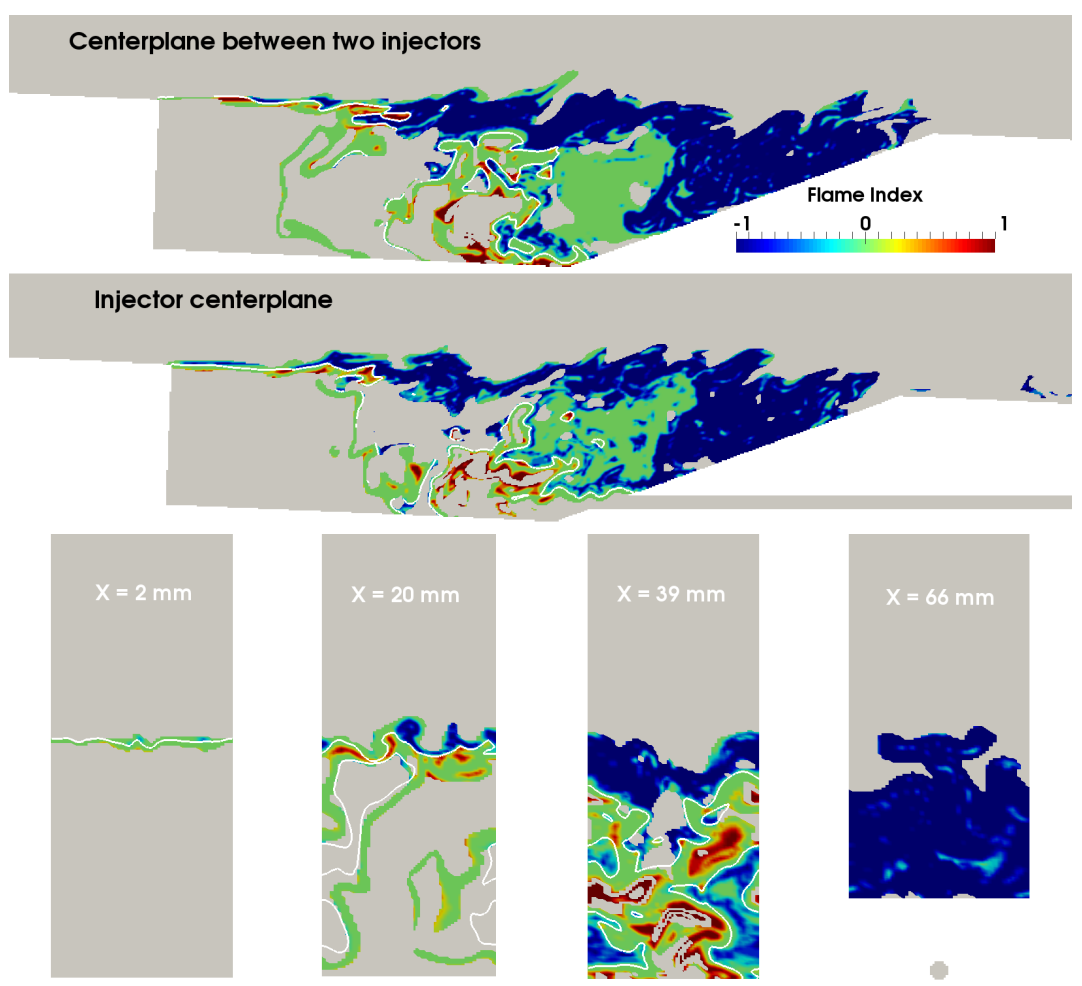

Flame Index

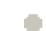

Figure 8.: Instantaneous flame index in the centerplane between two injectors, in the injector centerplane and in four planes with constant $x$ in the cavity. -1: lean premixed, 0: nonpremixed, 1: rich premixed. The isoline $Z=Z_{s t}$ is displayed with a white line. Case MF.

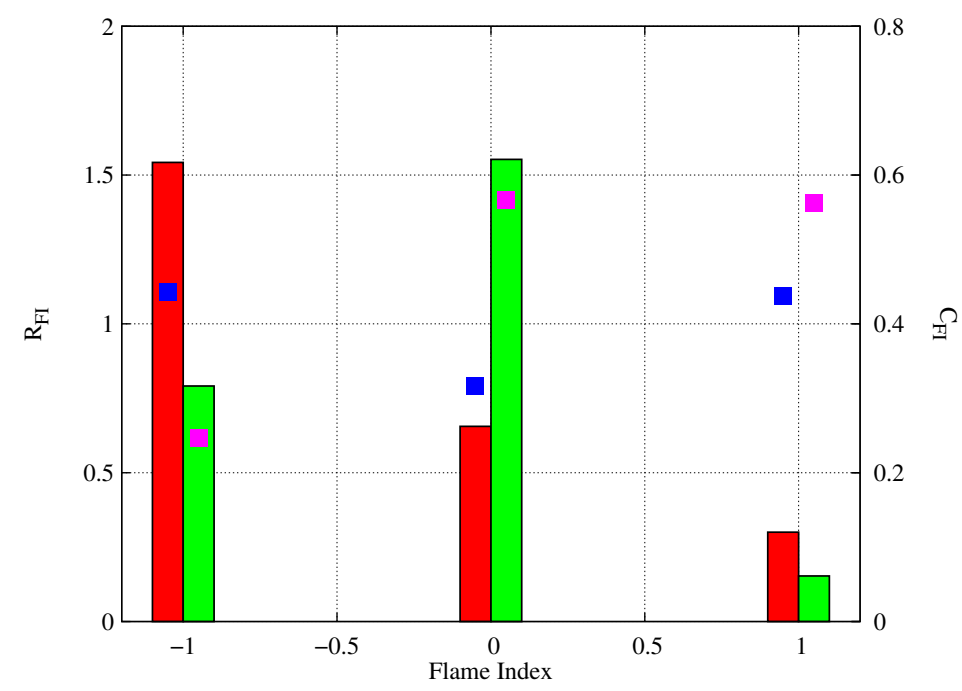

Figure 9.: Dimensionless conditional mean of heat release by flame index $R_{F I}$ (squares), and contribution of each value of flame index to the heat release $C_{F I}$ (histogram). Red and blue: MHF case, Green and pink: MF case. 
chemistry or a very coarse resolution implying a subgrid model for the filtered source terms were to be retained. Despite this change in combustion regime, a stable flame is still observed.

\subsection{Extinction mechanisms}

Starting with the stable case MF $(t=0)$, the fuel injection velocity is decreased up to 36 SPLM linearly within $0.5 \mathrm{~ms}$ in order to capture the extinction mechanism of the combustor. Once 36 SPLM have been reached, the simulation is left to evolve to track the extinction mechanism. In the procedure followed, it is not guaranteed that extinction occurs strictly at 36 SPLM but it is verified that, as in the experiment, this mass flow rate cannot sustain the stabilization of the flame.

The snapshots of heat release rate and $\mathrm{C}_{2} \mathrm{H}_{4}$ mass fraction are displayed in Fig. 10 for the injector centerplane. Instantaneous fields of $\mathrm{HRR}$ and $Y_{\mathrm{C}_{2} \mathrm{H}_{4}}$ at the centerplane between two injectors are in appendix A A transitional regime, where the successive velocity damping leads to combustion inside the injector, is observed up to $t=2 \mathrm{~ms}$. Contrary to the cases MHF and MF, the fuel is not able to reach the middle of the cavity and ethylene is immediately consumed at the exit of the injector. Combustion at the mixing layer is only fed by the initial huge stock of fuel at the front of the cavity which is starting to be cleared out. The lack of ethylene in the large recirculation zone causes a fuel shortage at the rear of the cavity, where the air entering is not mixed anymore with burnt products leading to a cooling of the cavity. At the next instants $t=4 \mathrm{~ms}$, ethylene is still burning at the exit of the injector, and the spare fuel at the cavity front has almost totally been consumed. The mixing layer between the freestream and the cavity is not anymore fed by the fuel from the small recirculation zone, so combustion vanishes in this region and the airflow entering the cavity cannot be heated. The reaction zone observed at the exit of the injector begins to decrease because the air coming from the main airflow is now cold and the reaction zone cannot resist anymore to the strong stretching.

\section{Conclusion}

The Large-Eddy Simulation of the AFRL supersonic cavity revealed the difference in combustion regimes when the incoming fuel flow was changed. At medium-high flow rate, the three combustion regimes, i.e. lean or rich premixed then non-premixed, are present in the recirculation zone which maintains the flame in the cavity. At medium flow rate, lean premixed and non-premixed flames are clearly observed in the main recirculation zone. For these two cases, the scenario of flame stabilization is the same: unburned fuel supplies a small recirculation zone where hot products are trapped; this stock of fuel then reacts with the incoming air which is more or less preheated depending on the flow of fuel injected. However, if the fuel flow decreases further, up to 36 SLPM, the fuel stock located in the small recirculation zone begins to decrease, limiting the auto-ignition of the mixture layer. Consequently, the energy released by combustion is less, leading to a drop in temperature in the main recirculation zone. There is therefore no longer enough energy to maintain combustion and the flame disappears.

\section{Acknowledgments}

The present work has been supported by the French Department of Defence (DGA). Computations ressources were provided by CRIANN and GENCI (allocation 2018-020152). 

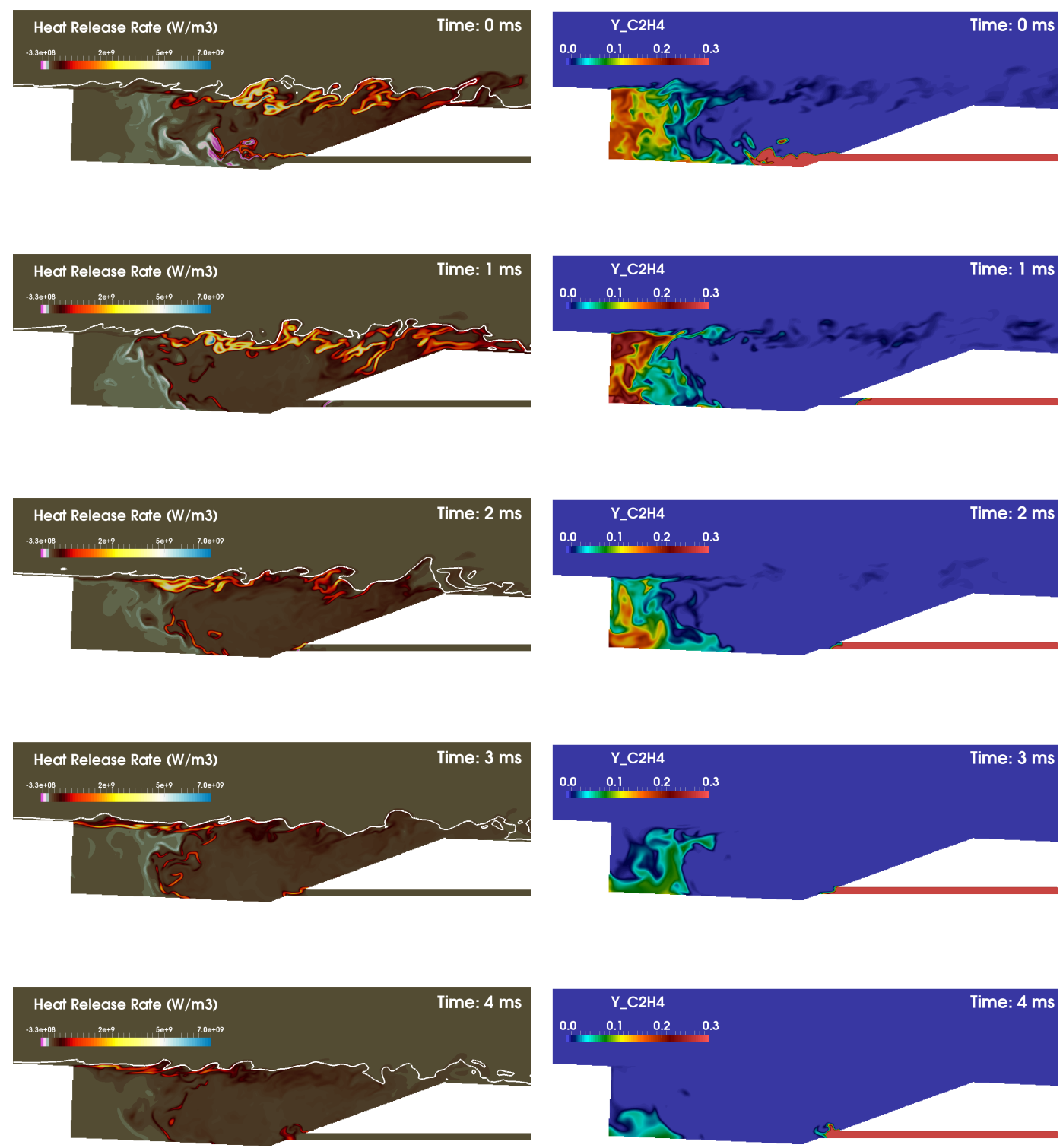

Figure 10.: Snapshots of heat release rate (left) and $\mathrm{C}_{2} \mathrm{H}_{4}$ mass fraction (right) at injector centerplane for a lean fuel loading, presented every ms from $t=0$ to $4 \mathrm{~ms}$.

\section{References}

[1] C. Segal, The scramjet Engine, Cambridge University Press, 2009.

[2] J. Urzay, Supersonic combustion in air-breathing propulsion systems for hypersonic flight, Annu. Rev. Fluid Mech. 50 (2018) 593-627.

[3] A. Ben-Yakar, R. Hanson, Cavity flame-holders for ignition and flame stabilization in scramjets: An overview, J. Propul. Power 17 (4) (2001) 869-877.

[4] F. Barnes, C. Segal, Cavity-based flameholding for chemically-reacting supersonic flows, Prog. Aerospace Sci. 76 (2015) 24-41.

[5] E. Gonzalez-Juez, A. Kerstein, R. Ranjan, S. Menon, Advances and challenges in modeling high-speed turbulent combustion in propulsion systems, Prog. Energy Combust. 
Sci. 60 (2017) 26-67.

[6] M. Berglund, F. C., Les of supersonic combustion in a scramjet engine model, Proc. Combust. Inst. 31 (2) (2007) 2497-2504.

[7] A. Saghafian, L. Shunn, D. A. Philips, F. Ham, Large eddy simulations of the hifire scramjet using a compressible flamelet/progress variable approach, Proc. Combust. Inst. 35 (2015) 2163-2172.

[8] L. Bouheraoua, P. Domingo, G. Ribert, Large eddy simulation of a supersonic lifted jet flame: Analysis of the turbulent flame base, Combust. Flame 179 (2017) 199-318.

[9] A. Vincent-Randonnier, V. Sabelnikov, A. Ristori, N. Zettervall, C. Fureby, An experimental and computational study of hydrogen-air combustion in the LAPCAT II supersonic combustor, Proc. Combust. Inst. 37 (2019) 3703-3711.

[10] J. Ruan, P. Domingo, G. Ribert, Analysis of combustion modes in a cavity based scramjet, Combust. Flame 215 (2020) 238-251.

[11] R. Miller, J. Foster, Survey of turbulent combustion models for large-eddy simulations of propulsive flowfields, AIAA J. 54 (10) (2016) 1-17.

[12] J. Ruan, L. Bouheraoua, P. Domingo, G. Ribert, Simulation of a scramjet combustor: A priori study of thermochemistry tabulation techniques, Flow Turbul. Combust. (accepted) DOI: https://doi.org/10.1007/s10494-020-00184-4.

[13] S. G. Tuttle, C. D. Carter, K.-Y. Hsu, Particle image velocimetry in a non-reacting and reacting high-speed cavity, J. Propul. Power 30 (13) (2014) 576-591.

[14] H. Do, C. D. Carter, Q. Liu, T. Ombrellow, S. Hammack, T. Lee, K.-Y. Hsu, Simultaneous gas density and fuel concentration measurements in a supersonic combustor using laser induced breakdown, Proc. Combust. Inst. 35 (2) (2015) 2155-2162.

[15] E. Hassan, D. Peterson, K. Walters, E. Luke, Dynamic hybrid reynolds-averaged navierstokes/large-eddy simulation of a supersonic cavity, J. Propul. Power 32 (6) (2016) $1343-1352$.

[16] R. A. Baurle, Hybrid reynolds-averaged / large eddy simulation of a scramjet cavity flameholder, AIAA J. 55 (2) (2017) 544-560.

[17] E. Hassan, D. Peterson, D. Walters, E. Luke, Dynamic hybrid reynolds-averaged navierstokes/large-eddy simulation of a supersonic cavity: Chemistry effects, J. Propul. Power 35 (1) (2019) 201-212.

[18] U. Guven, G. Ribert, Large-eddy simulation of supersonic $\mathrm{H}_{2} / \mathrm{O}_{2}$ combustion: application to a rocket-like igniter, J. Propul. Power 34 (2) (2018) 291-307.

[19] B. Duboc, G. Ribert, P. Domingo, Hybrid transported-tabulated chemistry for partially premixed combustion, Computers Fluids 179 (2019) 206-227.

[20] B. Duboc, G. Ribert, P. Domingo, Evaluation of chemistry models on methane/air edge flame simulation, Proc. Combust. Inst. 37 (2) (2019) 1691-1698.

[21] F. Ducros, V. Ferrand, F. Nicoud, C. Weber, D. Darracq, C. Gacherieu, T. Poinsot, Largeeddy simulation of the shock/turbulence interaction, J. Comput. Phys. 152 (2) (1999) 517-549.

[22] R. Swanson, E. Turkel, On central-difference and upwind schemes., J. Comput. Phys. 101 (2) (1992) 292-306.

[23] S. Tatsumi, L. Martinelli, A. Jameson, Flux-limited schemes for the compressible Navier-Stokes equations., AIAA J. 33 (2) (1995) 252-261.

[24] R. Swanson, R. Radespiel, E. Turkel, On some numerical dissipation schemes., J. Comput. Phys. 147 (2) (1998) 518-544.

[25] X. Petit, G. Ribert, P. Domingo, G. Lartigue, Large-eddy simulation of supercritical fluid injection, J. Supercritical Fluids 84 (2013) 61-73.

[26] C. Merlin, P. Domingo, L. Vervisch, Immersed boundaries in large eddy simulation of compressible flows, Flow Turbul. Combust. 90 (1) (2013) 29-68. 
[27] M. Germano, U. Piomelli, P. Moin, W. Cabot, A dynamic subgrid-scale eddy viscosity model, Phys. Fluids 3 (7) (1991) 1760-1765.

[28] R. Bird, W. Stewart, E. Lightfoot, Transport Phenomena, John Wiley and Sons, NY, 1960.

[29] J. Hirschfelder, C. Curtiss, R. Bird, Molecular Theory of Gases and Liquids, John Wiley and Sons, NY, 1969.

[30] Z. Luo, C. Yoo, E. Richardson, J. Chen, C. Law, T. Lu, Chemical explosive mode analysis for a turbulent lifted ethylene jet flame in highly-heated coflow, Combust. Flame 159 (1) (2012) 265-274.

[31] J. Ruan, Large eddy simulation of supersonic combustion in cavity-based scramjets, Ph.D. thesis, INSA de Rouen Normandie, https://tel.archives-ouvertes. fr/tel-02924025(2019).

[32] C. Jachimowski, An analytical study of the hydrogen-air reaction mechanism with application to scramjet combustion, Tech. Rep. 2791, NASA (1988).

[33] W. Cabot, Large-eddy simulations with wall models, in: Annual Research Briefs, Center for Turbulence Research, Stanford Univ., 1995.

[34] D. Davis, R. Bowersox, Stirred reactor analysis of cavity flameholders for scramjets, AIAA Paper 97-3274, in: 33rd Joint Propulsion Conference and Exhibit, 1997.

[35] R. Fiévet, H. Koo, V. Raman, A. Auslender, Numerical investigation of shock-train response to inflow boundary-layer variations, AIAA J. 55 (9) (2017) 2888-2901.

[36] R. Fiévet, V. Raman, Effect of vibrational nonequilibrium on isolator shock structure, J. Propul. Power 34 (5) (2018) 1334-1344.

[37] D. Zhao, E. Gutmark, P. de Goey, A review of cavity-based trapped vortex, ultracompact, high-g, inter-turbine combustors, Prog. Energy Combust. Sci. 66 (2018) 4282.

[38] H. Yamashita, M. Shimada, T. Takeno, A numerical study on flame stability at the transition point of jet diffusion flames, Int. Symp. Combust. 26 (1996) 27-34.

[39] A. J. Lock, A. M. Briones, X. Qin, S. K. Aggarwal, I. K. Puri, U. Hegde, Liftoff characteristics of partially premixed flames under normal and microgravity conditions, Combust. Flame 143 (2005) 159-173.

[40] R. W. Bilger, S. H. Starner, On reduced mechanisms for methane-air combustion in nonpremixed flames, Combust. Flame 80 (1990) 135-149. 
Appendix A. Extinction mechanisms: snapshots of HRR and $Y_{C_{2} H_{4}}$ at the centerplane between two injectors
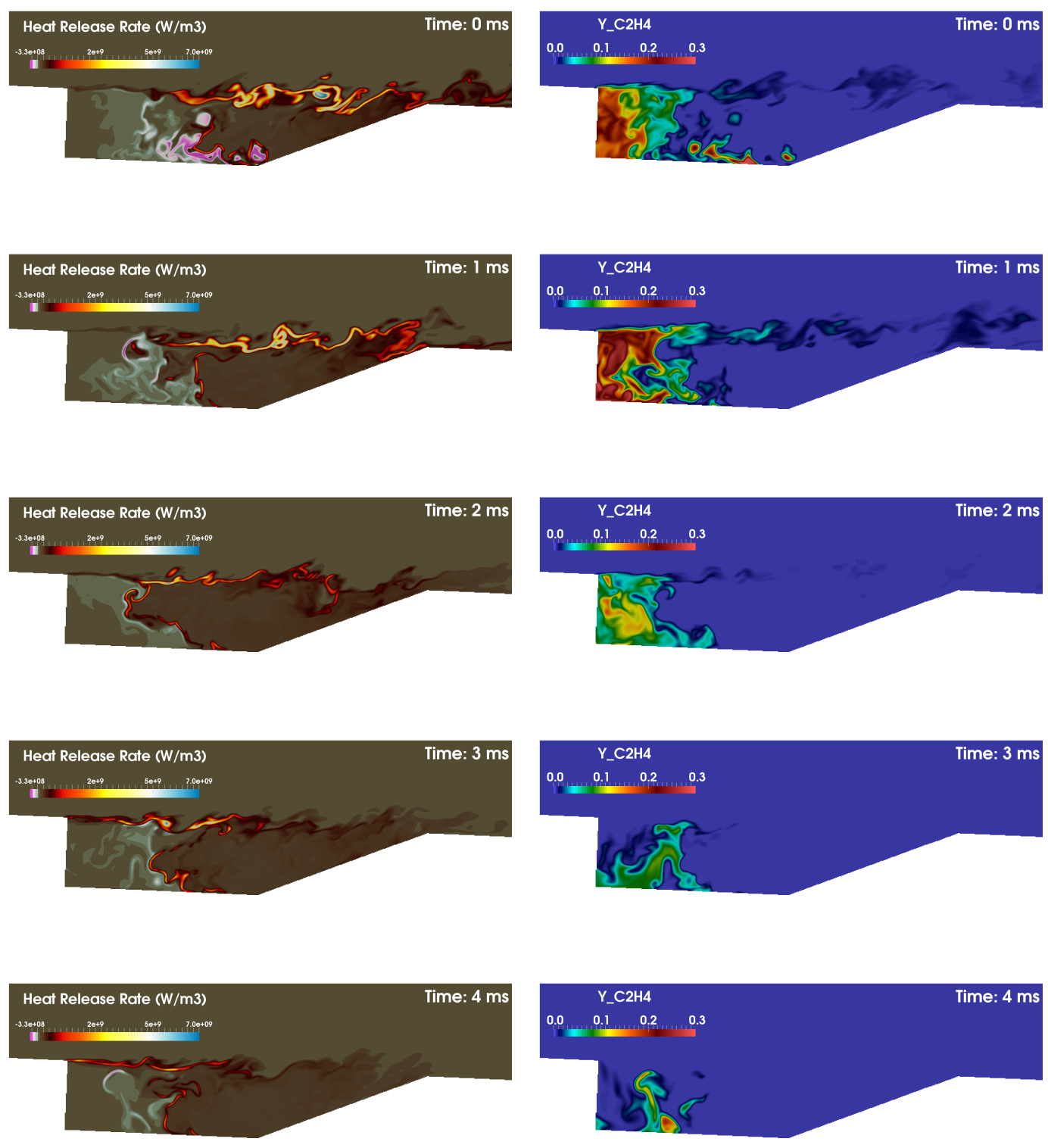

Figure A1.: Snapshots of HRR (left) and $Y_{C_{2} H_{4}}$ (right) at the centerplane between two injectors for a lean fuel loading, presented every $\mathrm{ms}$ from $t=0$ to $4 \mathrm{~ms}$. 